\title{
Vision deterioration in posterior reversible encephalopathy syndrome (PRES)
}

\author{
Dariusz Baranowski', Konrad Rejdak', Agnieszka Kiszka', Katarzyna Nowomiejska², Robert Rejdak² \\ 'Department of Neurology, Medical University of Lublin, Poland \\ ${ }^{2}$ Department of General Ophthalmology, Medical University of Lublin, Poland
}

\begin{abstract}
Posterior reversible encephalopathy syndrome (PRES) is a poorly understood, severe neurological condition secondary to impaired auto-regulation in the vertebrobasilar brain circulation resulting in subcortical vasogenic oedema distributed mainly in the white mater of the parietal and occipital lobes. Affecting optic radiation, PRES leads to visual deterioration, e.g. blurred vision, different types of vision field deficits, or even total blindness. The symptoms have a sudden onset and rapid progression, but are usually at least partially reversible. To bring this rare disorder closer to ophthalmologists, we present two cases of patients diagnosed with PRES.
\end{abstract}

KEY WORDS: vision deterioration, posterior reversible encephalopathy syndrome, posterior leukoencephalopathy syndrome, PRES

Ophthalmol J 2017; Vol. 2, No. 2, 61-67

\section{INTRODUCTION}

Patients with vision deterioration of sudden onset and fast progression need immediate management, including neuro-ophthalmological assessment. One of the possible causes of this condition is posterior reversible encephalopathy syndrome (PRES). Posterior reversible encephalopathy syndrome (PRES) is a poorly understood, severe neurological condition secondary to impaired auto-regulation in the vertebrobasilar brain circulation, resulting in subcortical vasogenic oedema distributed mainly in the white mater of the parietal and occipital lobes. In PRES, the development of vasogenic cerebral oedema affecting the white matter in the occipital and parietal lobes is essential [1]. It has various clinical manifestations, including severe headache, altered mental status with disorientation, changes in the level of consciousness, or generalised seizures [2]. Due to the high predilection of pathological lesions to localise in the occipital lobe white matter (affecting visual radiation), PRES manifests also as vision deterioration. To bring this condition closer to ophthalmologists, we introduce two cases of patients diagnosed with PRES.

\section{CASE PRESENTATION}

\section{CASE 1}

A 45-year-old woman with end-stage kidney disease secondary to glomerular nephritis (GN) was admitted to the Department of Neurology because of sudden-onset severe headache localised in the occipital area and rapidly progressing vision deterioration in both eyes (blurred vision). In physical examination, visual acuity of the right eye showed poor light perception, of the left eye - poor light perception, and the reaction of pupils to light was correct. Before admission, the patient was qualified to haemodialysis therapy for decreasing glomerular filtration rate resulting in hard-to-control hypertension (even using polytherapy with hypotensive drugs of 


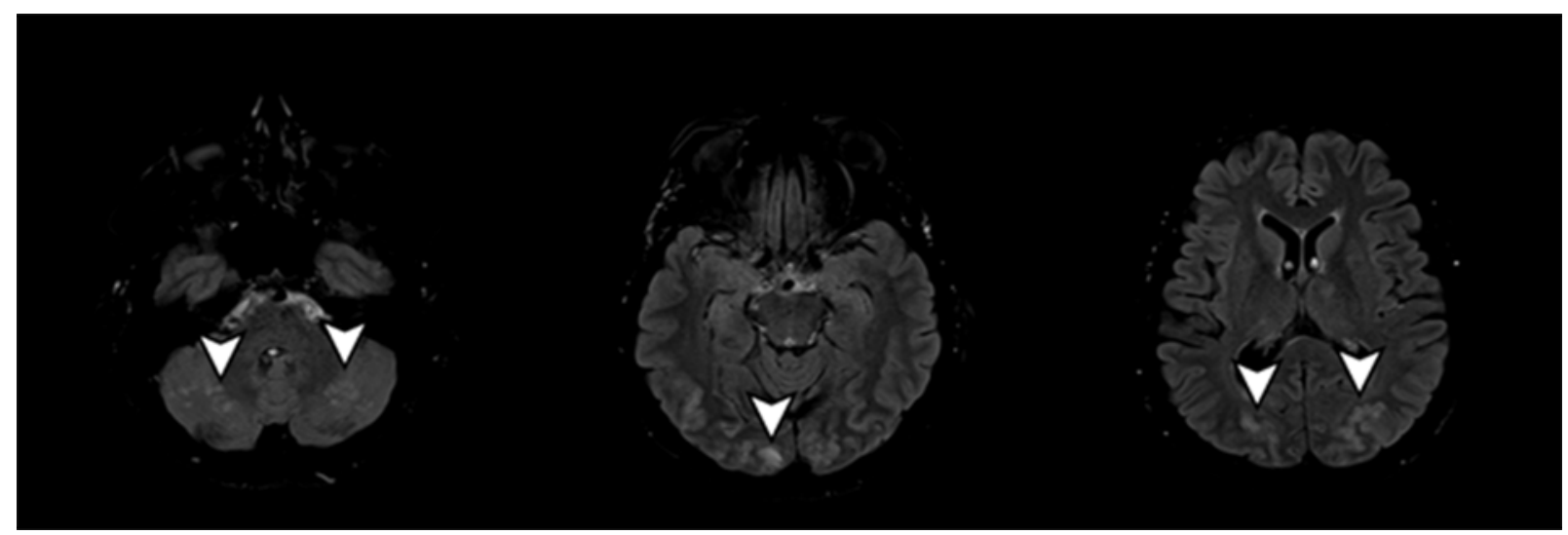

FIGURE 1. Patient 1 - MR T2 tirm hyper-intensive lesions in cerebellum and occipital lobes

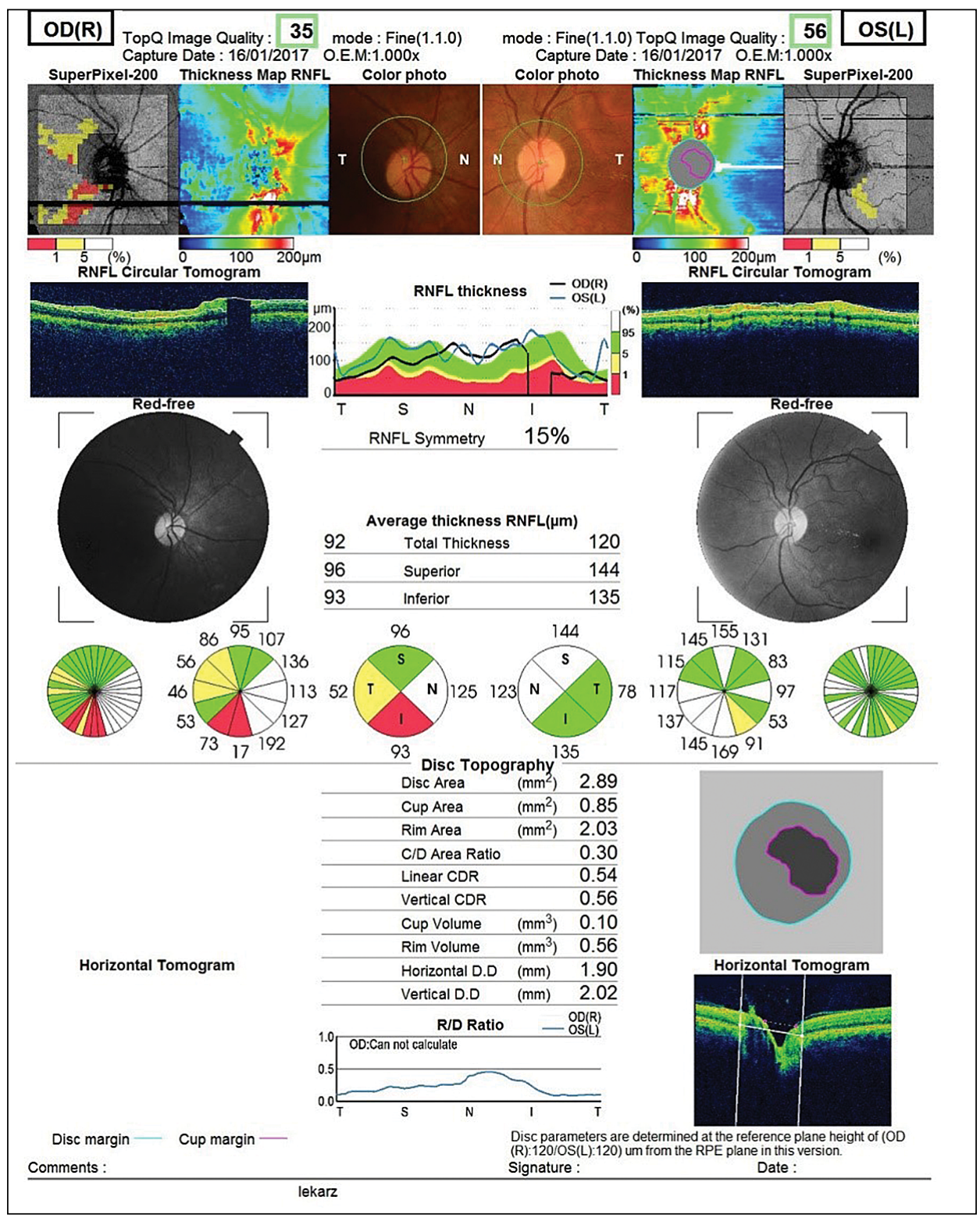

FIGURE 2. Patient 1 - result of ocular coherence tomography performed 8 weeks after an episode of posterior reversible encephalopathy syndrome (PRES) - increased peripapillary nerve fibre layer thickness in the right eye in the lower quadrant 


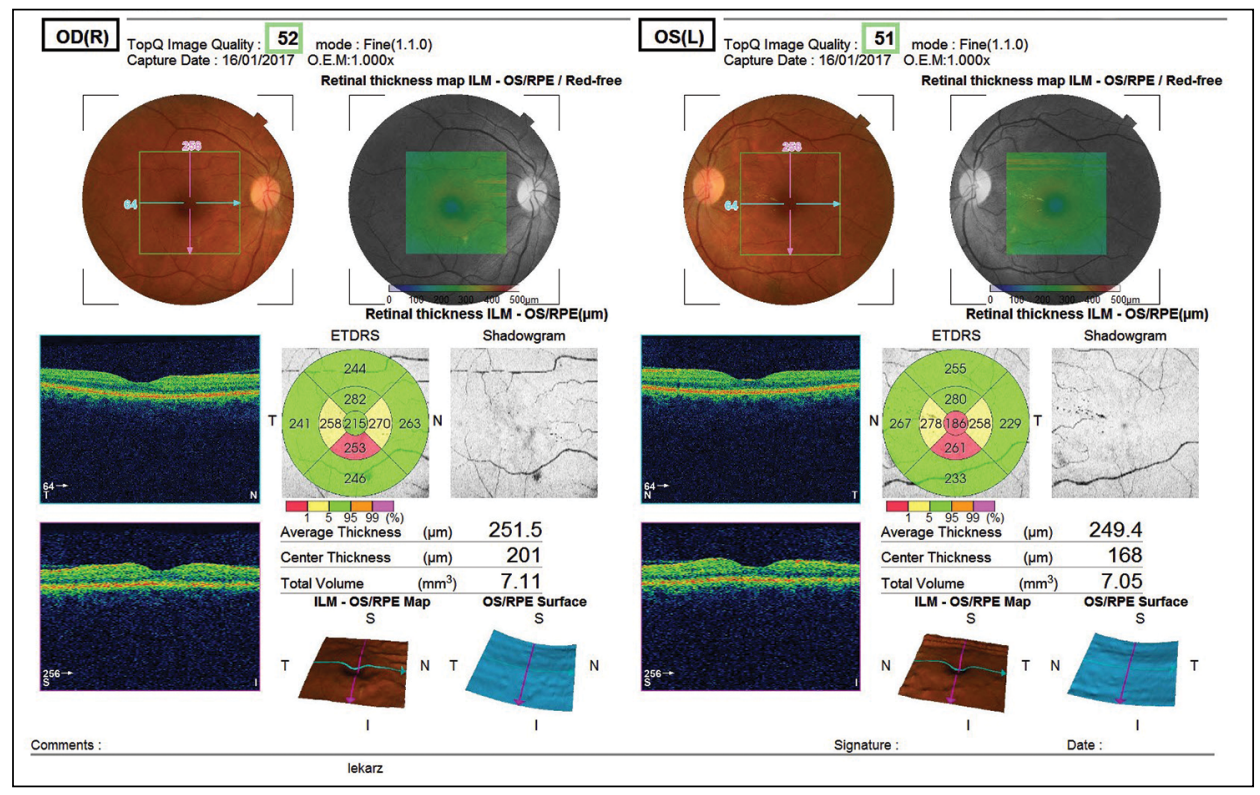

FIGURE 3. Patient 1 - result of ocular coherence tomography of the macula performed 8 weeks after an episode of posterior reversible encephalopathy syndrome (PRES) — normal profile in both eyes

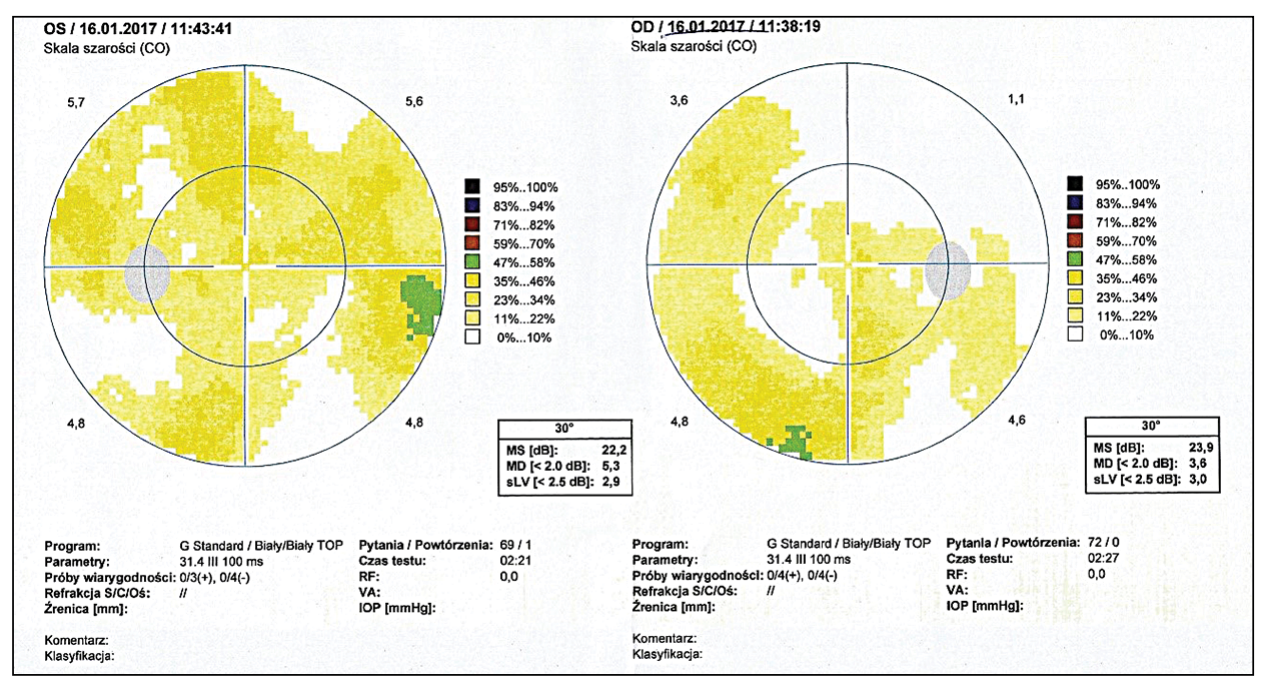

FIGURE 4. Patient 1 - visual field result of both eyes without abnormalities (examination performed after episode of PRES)

different mechanisms of action). MRI on admission revealed diffused hyper-intensive lesions in T2 and FLAIR (fluid-attenuated inversion recovery/FLAIR) sequences affecting the parieto-occipital brain cortex on both sides and the cerebellum (Fig. 1). During hospitalisation, her elevated blood pressure was normalised using continuous infusions of urapidil and loop diuretics under fluid and electrolyte (sodium and potassium) balance control. After discharge from the Department of Neurology, the patient was sent for haemodialysis. Eight weeks after hospitalisation in the Department of Neurology, visual acuity of the right eye was 1.0 without correction, of the left eye -0.8 without correction, and 1.0 with stenopeic correction. Fundus examination showed features of hypertensive angiopathy. Optical coherence tomography (OCT) showed a thickened peripapillary nerve fibre layer (RNFL) in the right eye; macular scans and visual field were normal (Figs. 2-4).

\section{CASE 2}

A 70-year-old female patient with uncontrolled hypertension was admitted to the Department of 


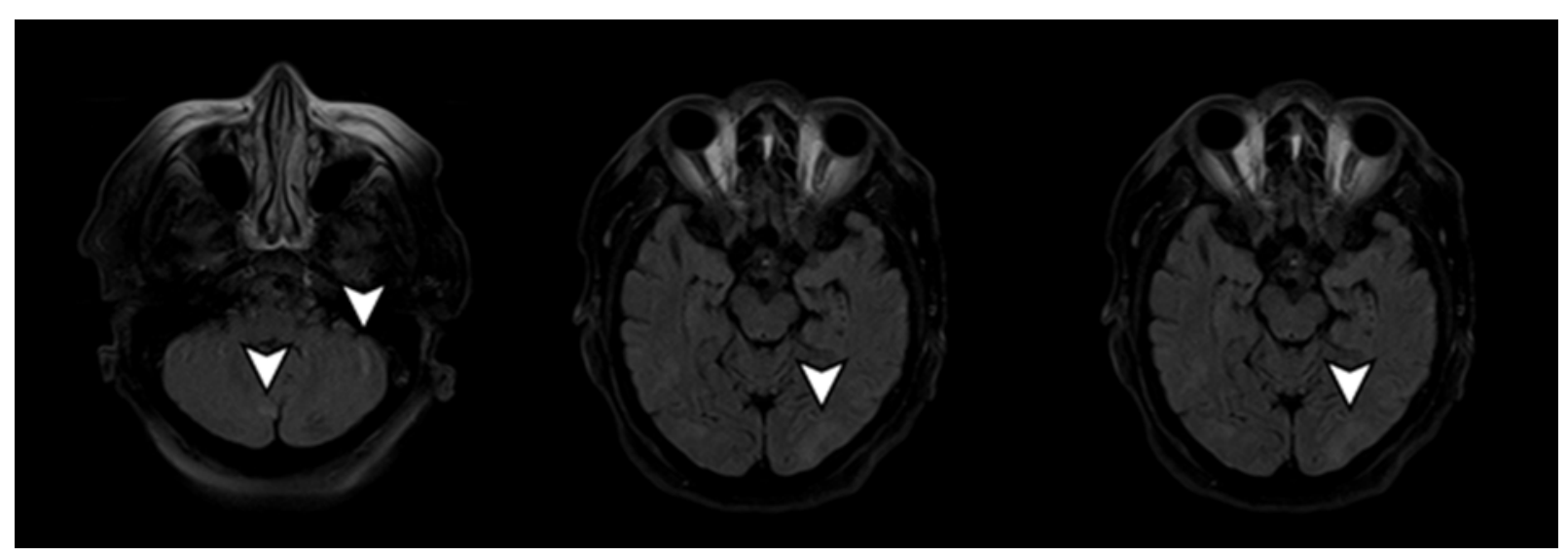

FIGURE 5. Patient 2 - MR T2 tirm hyper-intensive lesions in cerebellum and occipital lobes

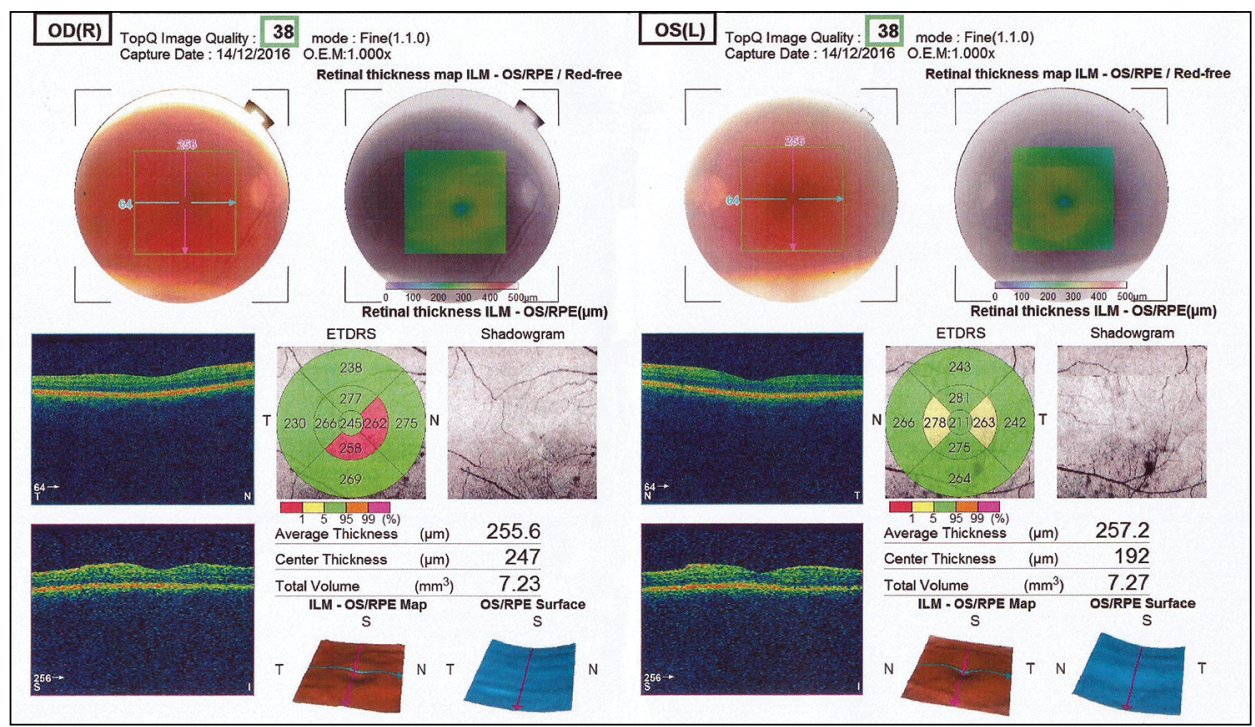

FIGURE 6. Patient 2 - result of ocular coherence tomography of the macula performed 2 weeks after episode of posterior reversible encephalopathy syndrome (PRES) — normal profile in both eyes

Neurology because of double eye total blindness, which occurred during hypertensive crisis. Routine CT did not reveal any structural brain pathologies; since an early phase of ischaemic stroke was suspected (early radiological signs of parenchymal ischaemia can be visualised by basic CT usually after few hours), the decision was made to amplify CT neuroimaging using contrast scanning with an angiography protocol to confirm or exclude occlusion of the arteries supplying the brain areas responsible for vision. The main cerebral arteries, including the posterior cerebral arteries on both sides, were not found to be occluded. Magnetic resonance (MR) imaging performed on the next day demonstrated gauzy (not well separated from the surrounding) hyper-intensive lesions in T2 and FLAIR sequences
(Fig. 5). Those areas were also characterised by moderate water diffusion restriction (diffusion-weighted imaging - DWI, and apparent diffusion coefficient - ADC sequences). To achieve stepwise normalisation of elevated blood pressure, intravenous continuous infusion of hypotensive drugs (urapidil) and intensification of oral hypotensive treatment were required. Next day the patient could see some light, and after two days of therapy she saw the profiles of individuals and objects. The fundus examination showed features of hypertensive angiopathy. Optic disk, blind spot, and retina were normal (Fig. 6-8). Follow-up MRI performed two weeks later showed complete reversion of pathological lesions.

During the control visit in the Department of General Ophthalmology, two weeks after hospital- 


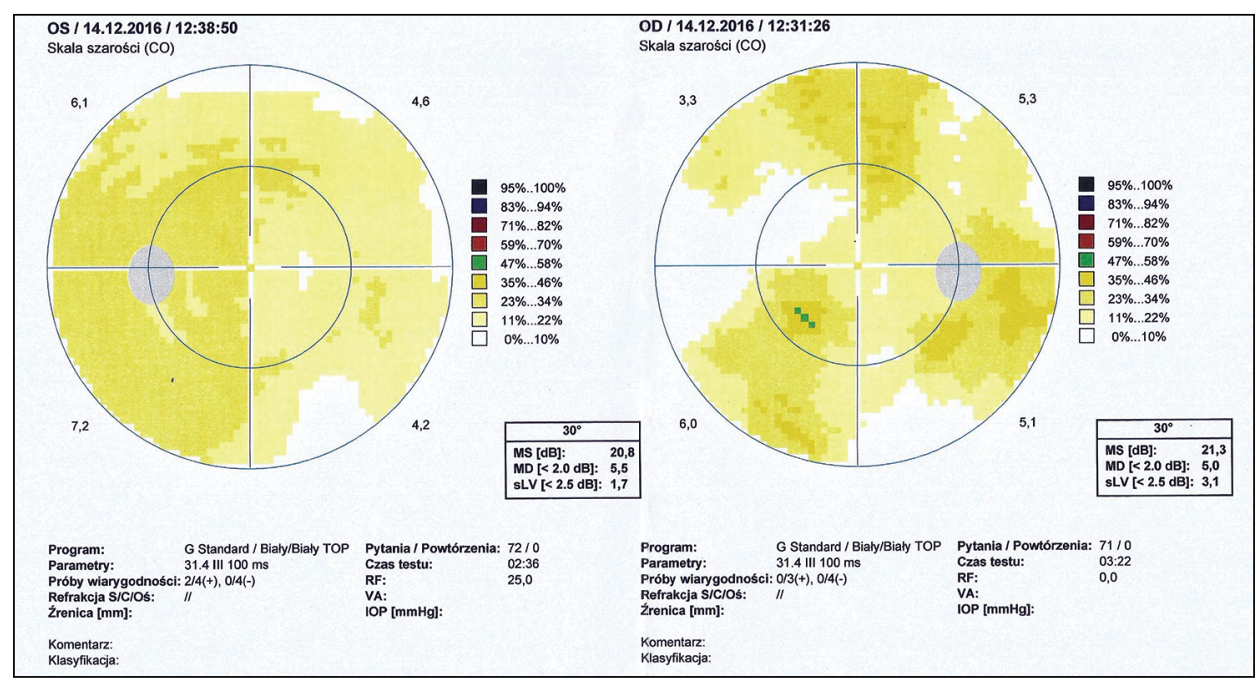

FIGURE 7. Patient 1 - visual field result of both eyes without abnormalities (examination performered after episode of PRES)

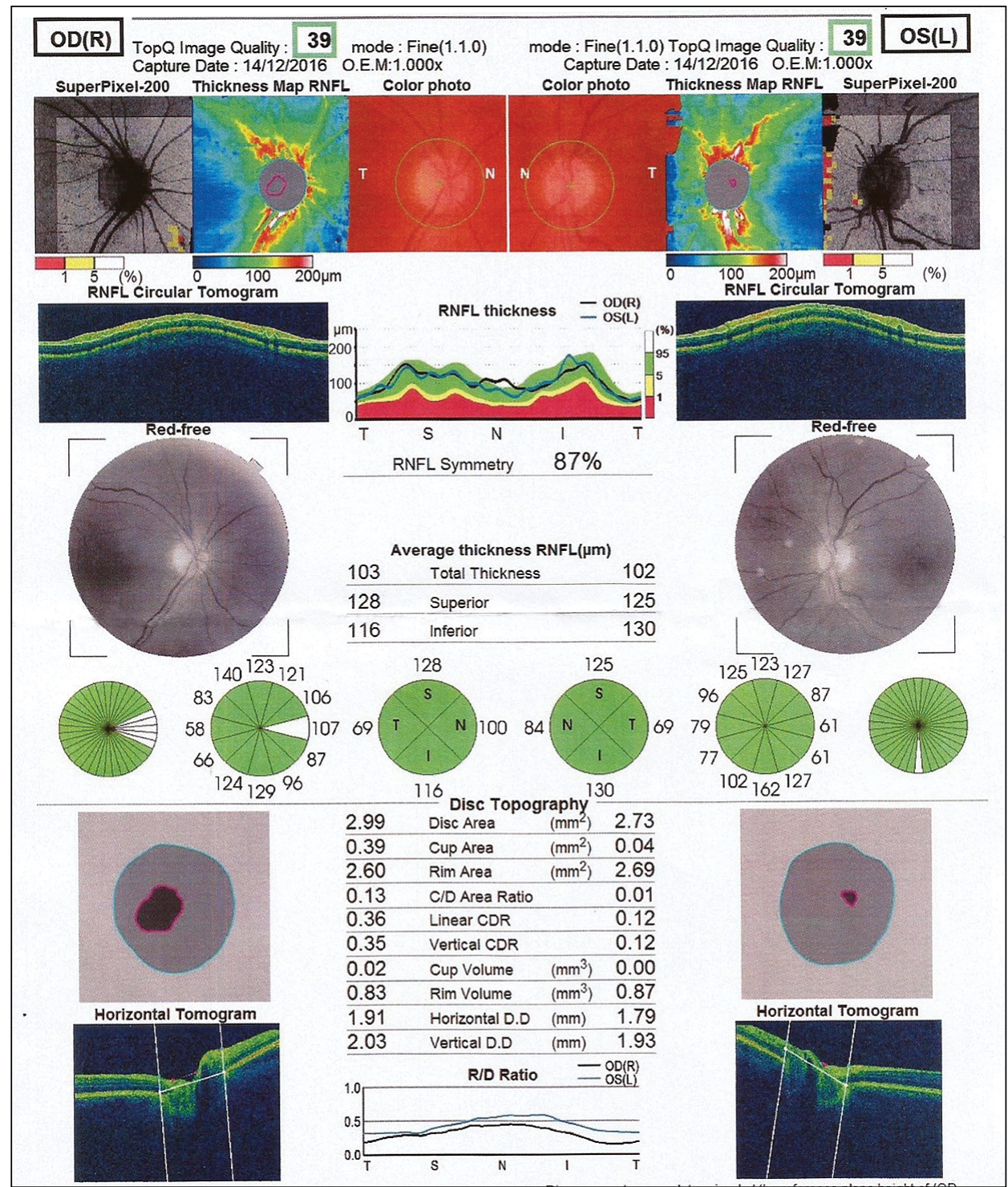

FIGURE 8. Patient 2 - result of ocular coherence tomography performed 2 weeks after an episode of posterior reversible encephalopathy syndrome (PRES) — normal peripapillary retinal nerve fibre layer in both eyes 
isation, visual acuity of the right eye was 0.3 with correction, and of the left eye -0.3 with correction. Only initial crystalline lens opacity and features of hypertensive angiopathy were found. OCT and visual field did not show any changes.

\section{DISCUSSION}

Posterior reversible encephalopathy syndrome (PRES) is considered a consequence of vasogenic cerebral oedema affecting the white matter in the occipital and parietal lobes. It is likely to be caused by poor autonomic innervation of arteries in the posterior brain circulation, resulting in their disadaptation to high systemic blood pressure. The unadjusted arteries allow the blood serum to soak into the brain parenchyma, called vasogenic oedema. This pathological mechanism can occur during hypertensive crisis in primary and secondary hypertension (connected with renal artery stenosis or end-stage kidney disease) or in pre-eclampsia $[2,4]$.

Another possible mechanism likely to lead to PRES in patients without elevated blood pressure is endothelial dysfunction. Highly specialised endothelial lining cells produce many different substances responsible for smooth blood streaming inside the arteries. Some of them, like prostacyclin or nitrogen oxide, cause dilatation of arteries. Endothelial damage-induced dysregulation can be caused by autoimmune diseases (including systemic vasculitis, e.g. Takayasu`s arteritis), malignant neoplasm, sepsis with multi-organ dysfunction, or adverse reactions to drugs (especially immunosuppressants and chemotherapeutics) [2]. The causes of PRES are presented in Table 1.

Posterior reversible encephalopathy syndrome (PRES) has various clinical manifestations, including severe headache, altered mental status with disorientation, changes in the level of consciousness, or generalised seizures [2]. Since the pathological changes have a specific predilection for the brain structures supplied by the vertebrobasilar circulation, posterior reversible encephalopathy syndrome usually (20-62.5\%) results in different types of visual deterioration: blurred vision, visual neglect, various types of vision field deficits (homonymous hemianopia), or even total blindness [2]. In the presented cases, visual loss was severe during the episode, but it was reversible, and at the end of the follow-up visual acuity was quite good - results of visual field examination and peripapillary RNFL were normal.

Every patient presenting with characteristic symptoms should undergo urgent MRI. In posterior reversible encephalopathy syndrome, MRI reveals the lesions that are hyperintensive in T2 and FLAIR sequences and localised in both sides (symmetrically) in occipital and posterior parts of parietal brain lobes $[1,2]$. In most cases, the changes persist for a few weeks. After that time, when the oedema descends, the follow-up MRI should be negative (in contrast to ischaemic stroke). Ophthalmological examinations in the acute phase (the first few days) can reveal optic disc swelling (papilledema) and haemorrhagic effusions in the eye bottom. Electroencephalography (EEG) during seizure attacks obviously shows epileptic-form patterns; during inter seizure intervals the basic electrical activity slows down to below normal.

Treatment depends on aetiology. The elevated blood pressure should be carefully treated using repeated slow doses or continuous infusion of short-lasting (easy to control effect) hypotensive drugs, e.g. labetalol or urapidil. If PRES is secondary to immunosuppressive therapy or chemotherapy, changes in drugs should be considered. Seizures should be managed with anti-epileptic drugs. If properly diagnosed and treated, PRES can be reversible.

Financial disclosure: none declared. Conflict of interest: none declared.

TABLE 1. Disorders and conditions considered to induce posterior reversible encephalopathy syndrome (PRES)

Primary and secondary (connected with renal artery stenosis or end-stage kidney disease) hypertensive crisis

Autoimmune diseases including systemic vasculitis (e.g. Takayasu's arteritis) and glomerular nephritis

Malignant neoplasm

Preeclampsia

Sheehan syndrome (pituitary gland infarct)

Sepsis with multi-organ dysfunction

Adverse drug reactions (especially immunosuppressant drugs) 


\section{REFERENCES}

1. Stevens CJ, Heran MKS. The many faces of posterior reversible encephalopathy syndrome. Br J Radiol. 2012; 85(1020): 1566-1575, doi: 10.1259/bjr/25273221, indexed in Pubmed: 23175479.

2. Thompson RJ, Sharp B, Pothof J, et al. Posterior reversible encephalopathy syndrome in the emergency department: case series and literature review. West J Emerg Med. 2015; 16(1): 5-10, doi: 10.5811/ westjem.2014.12.24126, indexed in Pubmed: 25671001.

3. Zhang Yu, Zhou J, Chen Yu. Posterior reversible encephalopathy syndrome in a child with steroid-resistant nephrotic syndrome: a case report and review of literature. Int J Clin Exp Pathol. 2014; 7(7): 4433-4437, indexed in Pubmed: 25120830.

4. Hammer ES, Cipolla MJ. Cerebrovascular dysfunction in preeclamptic pregnancies. Curr Hypertens Rep. 2015; 17(8): 64, doi: 10.1007/ s11906-015-0575-8, indexed in Pubmed: 26126779.

5. Hugonnet $E$, Da Ines D, Boby $H$, et al. Posterior reversible encephalopathy syndrome (PRES): features on CT and MR imaging. Diagn Interv Imaging. 2013; 94(1): 45-52, doi: 10.1016/j.diii.2012.02.005, indexed in Pubmed: 22835573. 